\title{
Research on Efficient Operation Mode of the Material Intensive of Power Grid Based on S\&OP
}

\author{
Yong-Huan HU \\ Purchase Department \\ State Grid Shanghai Municipal Electric Power \\ Company \\ Shanghai, China \\ E-mail: huyh@sh.sgcc.com.cn \\ Jun-Song WANG \\ Planning Department \\ State Grid Shanghai Procurement Company \\ Shanghai, China \\ E-mail: wangjunsong@ @sh.sgcc.com.cn
}

\author{
Wei ZHOU \\ Purchase Department \\ State Grid Shanghai Municipal Electric Power \\ Company \\ Shanghai, China \\ E-mail: zhou_wei@sh.sgcc.com.cn
}

\author{
Feng-Na DONG \\ QC Department \\ Shanghai JIULONG Enterprise Management Consulting \\ Co. Ltd. \\ Shanghai, China \\ E-mail: fengnadong@126.com
}

\begin{abstract}
This paper focuses on the issues of material intensive plan control and operation implementation under the mode of group purchasing. Starting with the analysis of the characteristics of demand differentiation, this paper also makes analysis of the characteristics of the efficient operation mode, the driving factors and also the connotation of the mode so as to determine the management needs, forming the key element of the formation of efficient operation mode. Using the advanced theories and methods of related supply chain management and S\&OP, from the perspective of demand chain and supply chain, using the improved system of optimization closed-loop method, this study builds the material intensive and efficient operation mode, which the main line is the "three systems "of "big plan" system of the whole supply chain with different demands, drive collaboration system and the mode operation performance evaluation system.
\end{abstract}

Keywords-material intensive; efficient operation mode; $S \& O P$ supply chain plan; collaborative strategy; performance evaluation

\section{INTRODUCTION}

Power grid enterprise resources intensive management from a strategic height, to strengthen the management of material resources, realize the enterprise resources economic gain, effective utilization of resources and the reasonable disposition, to resource integration and intensive control system as a whole and for the management of the main line, around the enterprise management innovation system which is formed by the material resources. Physical resource intensive management focus on centralized control and overall allocation of resources, the management scope covers front-end for source of supply chain procurement, back-end procurement execution, as well as the communication and liaison with external suppliers, is throughout the whole process of supply chain of integrated supply chain management system. More emphasis on physical resource intensive management to reach the company internal material resource value maximization, the optimization of their own resources at the same time, also to strengthen the role as the core enterprise in the supply chain, making a further strengthen the overall competitiveness of the supply chain.

And in the process of deepening physical resource intensive, material management to realize the advanced service grid system, strengthen the control, the process of power grid enterprise supplies factory, construction with its characteristics of modern logistics system, to strengthen and planning, finance, transport, construction management and other professional docking, promote business depth fusion, information sharing, in a concerted effort collectivize operation, ensure that all material intensive management measures put in place, the real achieve optimal allocation of system resources comprehensive and intensive, and efficient use of the sectional management material management to systematic operation, thus realize the coordinated and efficient operations of the vertical integration of transverse mode is very important.

\section{Challenges FACING}

Grid material supply chain management faces new challenges under the new situation the new normal:

(a) Surrounding the core of the focal point of the current material intensive system, put forward system operation pattern corresponding to promote improvement needs.

Resources currently facing intensive management system, both inside and outside of a lot of uncertainty, management of demand response "slow", "busy", "change" back-end supply problems, likely to cause uncertainty amplification system, system of instability increases, these are all influence the root cause of the physical resource intensive and efficient operation. 
(b) Compared with the requirements of the current company to speed up the innovation and development, materials and planning, investment, infrastructure, operations and other professional driving synergy between remains to be improved.

Grid of large-scale investment, high construction intensity, and a large number of new technologies, new products, new equipment have been put into use, material resources to the company's overall coordination ability, ability to support services and equipment safety quality put forward a new test. However, the material supply and demand side each major internal and external collaborative efforts is not enough, cause too much information not free, amplification, information sources such as information sharing problem, makes material side to the demand side and supply side of information control is weaker, thereby making it easier for the business operations in a busy, disorderly, passive situation.

(c) The plan execution control binding supplies side and the supply and demand side is not strong, the physical resource intensive and efficient mode of operation performance evaluation method system needs to be fully established.

In recent years, in the process of construction material resources intensive, actively promote supplies distribution center construction, supplier service hall construction etc., has preliminarily set up supplies of active service concept. But with the continuous development of material factory, company in material supply and demand planning control and coordinated response to the development and implementation, there are still some bottleneck problems in affected the physical resource intensive operation efficiency and operating performance, restricted the company material. To achieve the goal of intensive.

\section{THE RELEVANT THEORETICAL RESEARCH AND CASE APPLICATION OF ENLIGHTENMENT}

Through an analysis to the forefront of supply chain theory research, forming for grid resources intensive research and application of efficient operation mode has certain inspiration and reference.

\section{A. S\&OP Theory Research}

First meet the father of The S\&OP Dick Ling of S\&OP definition: "The process that enables a company both it's planning with the total company." Can divide three paragraphs to understand this concept, first of all, it is a process, the activities of the process is to plan, the task is to realize enterprise integration and coordination of plans.

S\&OP Process step see Figure 1
Figure 1. S\&OP Process step

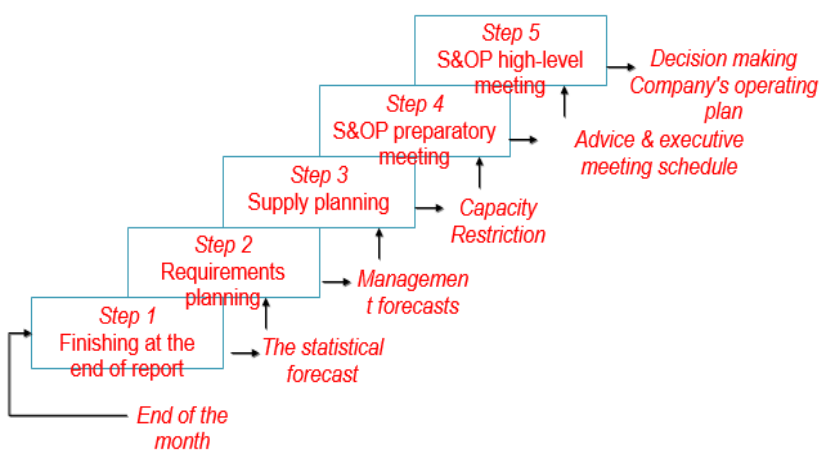

The process itself looks very simple, but process activity content is quite rich, involving the direction of the enterprise management and enterprise operation and management of multiple issues, including the formulation and implementation of medium and long-term plan, the balance of demand and supply, the implementation of the order fulfillment strategy, the adjustment of the inventory and service level, etc.

S\&OP as a management decision making and planning processes have been more than 20 years of practice, and in the near future to wide application and popularization of reason, is it maturity and effectiveness of the obtained widely recognized, On the other hand is its market orientation and the theory of dynamic balance in helping companies cope with various supply chain environment with excellent results.

Modern supply chain management practices will be accompanied by the market and competition environment of rapid change constantly deepen and popularity. In recent years, whether large or small and medium enterprises, the implementation of the S\&OP has become they improve the response capacity of the supply chain management practices.

We are all familiar with Dell beat rivals a winning formula is earlier than its competitors in terms of new products into the market for more than half a year's time, through the inventory, delay strategy, VMI and so on a series of management way, but the way behind an important and key management methods and planning - the integration plan, rarely get attention. It is interesting to note that the key factors are exalted S\&OP is it in order to perform the strategy of the implementation of the maturity and integration plan application, and help enterprises to improve market reaction ability effectively.

S\&OP system can help understand all of the supply and demand planning phase, including: collaborative demand forecasting, inventory efficiency, strategic planning, resource capacity planning, procurement and distribution plan, optimize the operation at the same time.

S\&OP theory to guide the MTO according to seasonal changes in demand, advantageous to realize the balanced production of suppliers, guide the MTS for inventory planning and forecasting, guiding plan - control - execution organization, process and mechanism design meeting. 


\section{B. $S \& O P$ Related Case Analysis}

(a) Based on Philip S\&OP project needs collaborative system

Philips Lighting main business for the big events such as Olympic Games, the expo will provide professional Lighting and decorative lamps and lanterns, has the characteristics of a typical project configuration type business. Project configuration type industry is highly complex and uncertain, although small number but customized product SKU configuration degree is very high, complex production process of raw materials are numerous; Bidding model, leading to the front almost no ready-made model can predict the market demand; Delivery speed after the high demand for the supply chain, procurement, production and other functions moment in the passive status of emergency response; Demand front-end and back-end supply collaborative S\&OP is almost a "Mission Impossible".

So how do you reconcile good "transparency", "reaction time" and "contradiction between company departments response"? One way is to change the S\&OP process, from one size fits all round a month to return to the sectional management in different stages of the S\&OP and response. Namely: the implementation of real-time updating of the project, in the end of the sales by web and mobile APP timely submit the project details. And collect and refine, and release to the appropriate department. Important information, such as high risk and project late stage change will cause related departments and even management attaché's importance to and dealt with the first time. Sales manager also through the tool to understand and manage a line sales progress.

Supply chain, procurement, engineering customization department in a week to deal with the future short-term (3 months, for example) update all items and arrange accordingly and feedback to the business department or arrange the necessary communication and discussion. Sales, marketing and management will also get the corresponding report has got the latest dynamic. Monthly summary by goalkeeper future medium (such as half a year or three quarters) of the data and discuss the trend of development, the market plan and supply plan, capacity plan, etc. This and ordinary S\&OP is more close to. Of course, the key and strategic projects tend to be the focus of the management discussion.

(b) The case on the application of the power grid enterprise resources intensive $S \& O P$ mechanism

(1) Project type business requirements often have a lot of uncertainty. Customers from the initial project to research, selection, screening, early and late bidding, all stages of the contract can be affected by many factors and produce change. Related to the government investment or associated projects in particular. Second, the project configuration type business product customization (non-standard) more often. Third, between the product form a complete set may need each other, and the construction progress of form a complete set, and service of form a complete set of...This than ordinary demand number + time + place of a dimension.

(2) Synergy technology engineering services supplies demand. How to solve the power of all kinds of engineering construction supplies supply and demand coordination technology services. How to prepare the demand planning, supply chain management department of supply chain management department how to set up and the demand side and supply side effective collaborative decision-making mechanism.

\section{GRID RESOURCES INTENSIVE AND EFFICIENT OPERATING MODEL BUILDING RESEARCH}

\section{A. Physical Resource Intensive And Efficient Operating Model Architecture}

Material supply chain management is for the entire supply chain planning and operating supplies (execution and implementation and activities in the process of collaborative), supervision, control and evaluation, optimization and improvement), analysis and decision of all kinds of activities and processes, Physical resource intensive, therefore, efficient operation mode to build from general plan system, the full link drive coordinated and comprehensive performance evaluation of three aspects carries on the design and implementation of the advance work, pay more attention to the integration of supply chain demand chain and supply chain plans as a whole, strengthen the control over material resources plan execution and rectifying, building material, across the line upstream and downstream coordination, formation mode optimization operation supervision, control and evaluation.

Through the establishment of the supply chain fully available plans - control - execution system, the implementation of the whole chain internal and external business efficient co-ordination, to form effective operating model to improve system performance evaluation, implement differentiation under the demand of efficient operation mode.

\section{B. Resources Efficient Operation Mode Of "Master Plan" System}

1) Big Plan System Definition and Implementation of The Key

Grid resources intensive and efficient operating mode of the program system is based on material intensification strategy and target, establish the planning and control, planning, implementation and control, project evaluation and evaluation system, promote large feedback continuously improve plan system, form a procurement plan - inventory replenishment planning, distribution planning, procurement execution - inventory replenishment perform - distribution, through the sharing information platform will demand chain plan, material management and supply chain plans to achieve seamless and integration, and promote the electrical material supply chain by using the method of performance evaluation of large improvement of the plan.

Grid resources intensive and efficient operation mode of big plans to implement the key:

(1) On the need of differentiation build plan different control methods.

(2) Before and after the implementation of supply chain planning fully available.

(3) establish a plan execution control standard and feedback mechanism. 
(4) Emphasis on the efficiency of material demand information.

Information real-time update project information according to the event trigger

Supplies demand along with the perfecting engineering process

Cycle synergy, regular monitoring and feedback

(5) Through big plan evaluation and examination form closed-loop management.

\section{2) The Implementation of A System of Big Plans}

\section{a) Large planning process requirements}

Form a unified specification material planning and dynamic adjustment of processes, standards, and the mechanism, strengthen the plan of dynamic adjustment and response capabilities; Formation of standards, processes and control method of controlling plan changes, reduce to plan execution control plan changes at random disorderly situation.

Through the implementation of main power supplies demand supply annual plan, quarterly plan, monthly plan, weekly supply plan execution control and change control processes, strengthening plan execution control.

Big front end demand chain plan including demand planning, material requirements planning and the arrival of the focus is the milestone/schedule node demand, according to the engineering demand plan, internal locus of control pour push the arrival of the goods according to the actual engineering situation, paying more attention to power grid construction materials production requirement, for the purpose of target reduction plan changes, improve delivery accuracy. Big backend supply chain plan including delivery capacity plan and drawings, production scheduling, factory acceptance, delivery plan. Supply chain plans to focus more on supplier capacity and production equilibrium is given priority to, aim to improve supplier productivity prediction and balanced. Grid all big plan through the implementation of the annual material demand forecasting, supply chain balanced purchasing batch plan arrangement, and then forecasts by implementing quarterly supply and supply capability assessment, implementation power grid construction materials demand from the supplier's production supply capacity balance as a whole.

Material requirements planning information with power grid construction progress update and accurate, and gradually achieve the progress of the scroll information update mechanism (see Figure 5 (see Figure 5)), to improve planning accuracy, perform corrective plan and response ability is very important.

Project units (material demand side) of the construction schedule, material requirements such as key information scroll updates, step by step on supplies of materials provided by the demand side to monitor project progress and material needs such as key information and early warning.

\section{b) The big plan operation mechanism}

Improve the precision of the program, the realization of the execution of rectifying unceasingly, on the basis of plan anticipation sex, implement the S\&OP mechanism under normal condition, in the case of supply and demand side move, and start under the abnormal state of S\&OP mechanism and the decision-making process.

Plan subject dynamic adjustment

(1) to follow up the project of the key date: along with the advancement of construction schedule, early cuts through demand and follow up, continuously updated engineering key date, reasonable to better determine the goods start time.

(2) According to the progress of the situation of material dynamic adjustment plan elements: according to the progress of the dynamic adjustment and the project schedule change, analysis may cause potential uncertainty and changes to materials, promote the dynamic adjustment of material plan and to increase the ability to respond.

(3) The annual demand for engineering and to predict the concentration in certain period of time in the future. Demand for engineering materials in the future a certain period of forecast, demand for supplies in certain period of time and concentration of anticipation, enhance predictability and anticipation ability of the plan.

Plan subject to handle change

(1) For engineering changes dynamically adjust or beyond the reasonable range of purchasing and supply cycle response, because goods can't meet the plan as it is belong to the big plan changes, this kind of situation need supplies side and demand side to form a coordinated plan as a whole. A final decision results is based on standard process adopts the material purchasing supply emergency channel meet the demand of engineering materials, the second is the progress according to the material purchasing and supply nodes for adjustments, held a meeting to be led by a resolution plan subject, and to adjust plan. This is a material between side and demand side as a whole the decision-making process.

(2) Major change supplies demand purchasing application decisions directly lead to large changes in implementing the plan. Demand for units to delete order or order has not been implemented, to delete the order or order change to carry out project construction unit and the process of examination and approval, and carries on the communication and coordination with the supplier, formed as a whole after the adjustment plan.

\section{Resources Efficient Operating Model Driven Collaborative System}

On the basis of the theory of supply chain collaboration, first determine synergy value target, the key factor analysis model, starting from the business level, combing the key elements of the impact on goal, will implement the factors of success to the corresponding business processes, business process decomposition, identifying synergy point, using the collaborative analysis model, detailed analysis of coordination point (constraints, key factors and control methods, etc.), set the collaborative strategy, standards, and mechanism, implement and evaluate the business synergy effect.

From business level, the comb materials and construction schedule, supplier production key nodes, and combing the key elements of the impact on goals, combined with the project process and materials for all links, on demand, material side chain and supply chain to identify key elements together. 


\section{Resources Efficient Operation Mode of Performance Evaluation System}

a) Efficient Operating Model Performance Evaluation Index System Design

Supply chain SCOR model from a reliability, responsiveness, flexibility, costs, assets, five aspects of supply chain performance measurement and evaluation, and $11 \mathrm{KPI}$ index are put forward. At the same time, considering the power efficient operation mode for the procurement and supply process emphasized the prospective planning and material purchasing supply work balance, thus increased the forward-looking and proportionality indexes. And because the asset and the cost is belong to the category of power supply enterprise goods and materials in the supply chain finance, will also be merged. In addition, due to the types of power supplies, and a lot of need, to collaborative supply chain demand is high, at the same time supply is in order to ensure normal construction and operation of power grid. Therefore increased the effective operating model big plans collaborative indexes.

Eventually form efficient operation mode based on SCOR model performance evaluation index system, from the reactivity, reliability, flexibility, collaborative, prospective, balance, cost/assets in seven aspects of power efficient operations performance measurement and evaluation, and proposes 17 KPIs.

\section{b) The Index System of Application}

Performance evaluation system of the application process see Figure 2.

Figure 2: Performance appraisal system application process

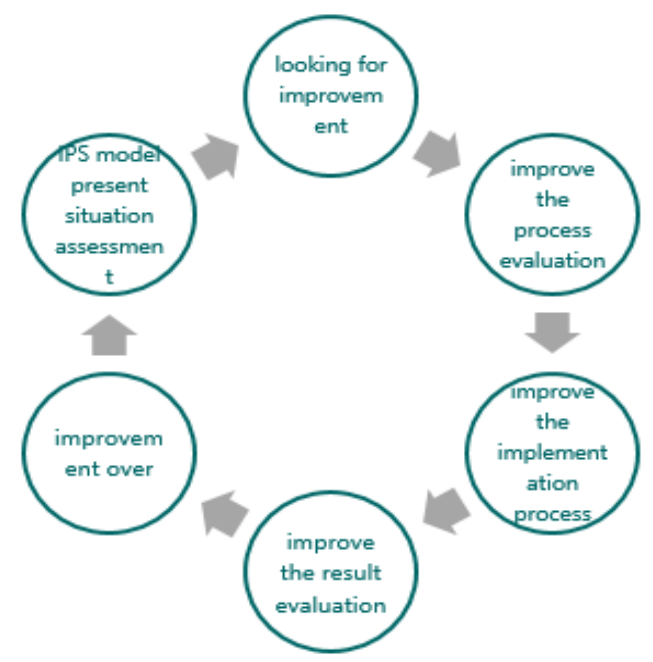

1. Situation assessment: By applying power efficient operation mode operating performance evaluation index system of present situation analysis, find out poor indicators, further subdivided into the business layer and the index, find the problems of business process, and also is the point of improvement.

2. Process evaluation: After find improvement point it needs to be aimed at the improvement of some specific process improvement measures, in the process of specific measures to implement, to constantly evaluating indicators of the process, monitor the implementation process, to ensure that the measures in place.

3. The results of assessment: Process improvement implementation is completed, need to once again to the whole supply chain performance evaluation, comparing with the results before evaluation, to test process improvement effect, can be set according to the improved condition after system target, and then to model performance evaluation, this process repeated cycle.

\section{SUMMARY}

By establishing control - oriented differentiation demand big plan execution system, strengthen the related parties of the supply chain drive together, form a comprehensive performance evaluation method system, construct a differentiated demand driven by intensive system efficient operation mode of material resources, to achieve efficient, collaborative, adaptive system of material resource intensive, improve overall operating performance of supply chain is of great significance.

\section{REFERENCE}

[1] Nairu Xu, Jiabao Liu, Binghua Yuan. Agile Supply Chain Management Research, Journal of Chongqing University (social science edition) [J],2014,(6):62-70

[2] Guozhu Jia, Chengyan Zhang.Agile supply chain agility analysis, industrial engineering [J],2006,(4):7-11

[3] Shoufeng Zhang, Jintian Ge, Minmei Huang. On building the flexible supply chain strategic thinking, scientific and technological progress and countermeasures [J],2004,(1):112-113

[4] Yuansheng Li, Feifan Ye, Zhimei Fang, Jianguo Yang. Flexible supply chain optimization and suppliers response time analysis, industrial engineering and management $[\mathrm{J}], 2005,(1): 89-93$

[5] Dongmei Ni, Qiuhong Zhao, Haibin Li. Comprehensive demand forecasting model and its integration with inventory decision-making research, journal of management science [J],2013,(9):44-52

[6] Zhiqing Zhang. Facing the demand uncertainty of supply chain collaborative demand forecasting research [D], Harbin institute of technology, management science and engineering,2010

[7] Yulan Tan, Xiaoyan Yang. Eliminate the strategic analysis of supply and demand uncertainty of supply chain, international business research $[J], 2008,(6): 59-62$ 